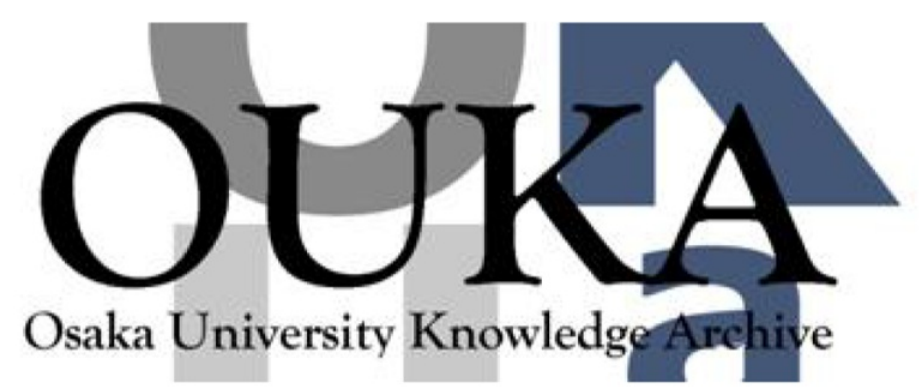

\begin{tabular}{|c|l|}
\hline Title & $\begin{array}{l}\text { Control of the bias tilt angles in nematic } \\
\text { liquid crystals }\end{array}$ \\
\hline Author(s) & $\begin{array}{l}\text { Yablonskii, S. V. ; Nakayama, K.; Okazaki, S. et } \\
\text { al. }\end{array}$ \\
\hline Citation & Journal of Applied Physics. 85(5) p. 2556-p. 2561 \\
\hline Issue Date & $1999-02-19$ \\
\hline oaire:version & VoR \\
\hline URL & https://hdl. handle. net/11094/75853 \\
\hline rights & \\
\hline Note & \\
\hline
\end{tabular}

Osaka University Knowledge Archive : OUKA

https://ir. Library. osaka-u. ac. jp/

Osaka University 


\section{Control of the bias tilt angles in nematic liquid crystals}

Cite as: Journal of Applied Physics 85, 2556 (1999); https://doi.org/10.1063/1.369574

Submitted: 06 April 1998. Accepted: 19 November 1998. Published Online: 19 February 1999

S. V. Yablonskii, K. Nakayama, S. Okazaki, M. Ozaki, K. Yoshino, S. P. Palto, M. Yu. Baranovich, and A. S. Michailov

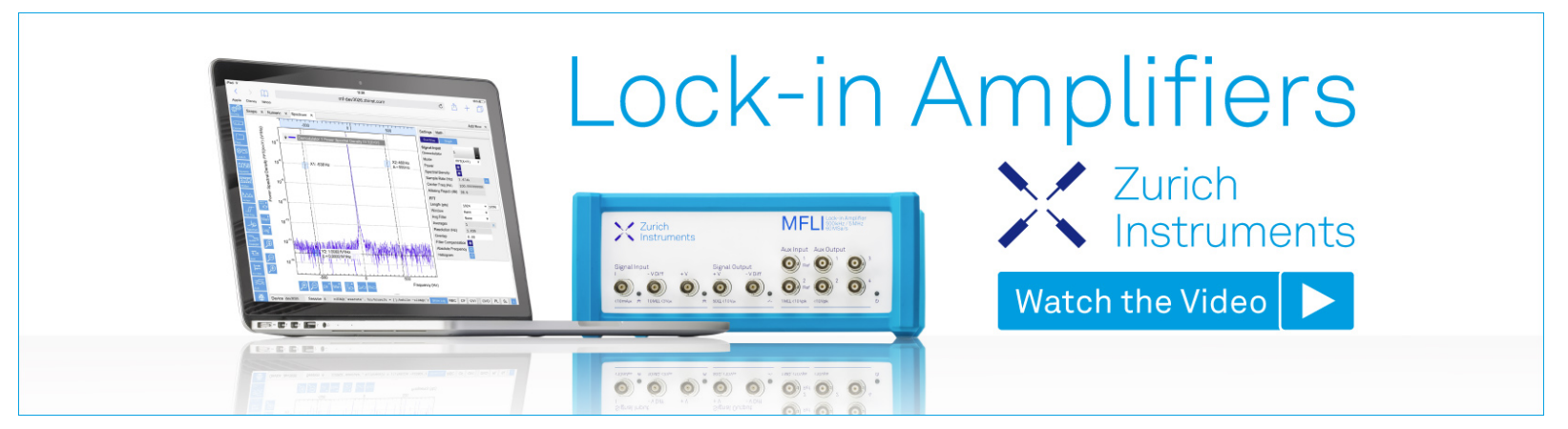




\title{
Control of the bias tilt angles in nematic liquid crystals
}

\author{
S. V. Yablonskii, ${ }^{\text {a) }}$ K. Nakayama, S. Okazaki, M. Ozaki, ${ }^{\text {b) }}$ and K. Yoshino \\ Department of Electronic Engineering, Faculty of Engineering, Osaka University, 2-1 Ymada-Oka, Suita, \\ Osaka 565-0871, Japan
}

\author{
S. P. Palto, M. Yu. Baranovich, and A. S. Michailov \\ Institute of Crystallography of RAS, 117333, Leninskii pr.59, Moscow, Russia
}

(Received 6 April 1998; accepted for publication 19 November 1998)

\begin{abstract}
The pretilt angle controlled by electric field was studied by the modulation ellipsometry technique. The easy direction of compensated nematic liquid crystals was controlled by surface flexoelectric torque created by the linear coupling of the director deformation and electric field. The weak anchoring energy necessary for the occurrence of flexoelectric distortion was produced by unidirectional rubbing of the clean indium-tin-oxide covered glasses with a cotton cloth. The pretilt angle was measured as a function of electric field. Long relaxation times of the optical response (hundreds of seconds) were observed. The rubbed thin polyvinyl alcohol and polyimide aligning layers were seen to promote strong anchoring energy $\left(>0.5 \mathrm{erg} / \mathrm{cm}^{2}\right)$ preventing any deviation of pretilt angle and, consequently, to suppress the optical response. The probable applications of the obtained results are discussed. (C) 1999 American Institute of Physics.
\end{abstract}

[S0021-8979(99)08404-2]

\section{INTRODUCTION}

The pretilt bias angle, defined as director inclination to the boundary surface is one of the most important parameters of liquid crystal displays. In fact, the pretilt angle is a parameter reflecting complex interactions of the solid substrate with the liquid crystal. The optimized bias tilt angle essentially improves the electrooptical properties of liquid crystal devices such as viewing angle, ${ }^{1}$ response time, ${ }^{2}$ steepness of the optical transmission-voltage curve, ${ }^{3}$ and so on. Various methods employed for the surface alignment of liquid crystals (LCs) such as rubbing of thin polymer films, ${ }^{4}$ oblique evaporation of $\mathrm{SiO}^{5}$ and photoalignment ${ }^{6}$ provide very effective orientation of LCs with definite static pretilt angles. Of special interest are the "command surface effects" 7,8 where the pretilt angle is controlled by the in situ photoexcitation of the aligning surface layers, for example in an assembled cell.

Recently, we have shown experimentally ${ }^{9-11}$ that orientational waves can be excited due to the linear flexoelectric coupling between a nematic LC (NLC) and an externally applied sinusoidal electric field. The flexoelectric torque arises in the boundary layers and slightly disturbs the equilibrium position of the pretilt angle, thus producing elastic orientational waves. The dispersion relation of the excited waves is of a diffusive type, so that large amplitude waves appear at the boundary near the exciting electrode, where they can be detected by an ellipsometric technique.

In the present work we have modified this experimental approach using an asymmetric driving voltage which allows simultaneous control and measurement of the pretilt angle. Thus, our attention is focused on the measurement of the

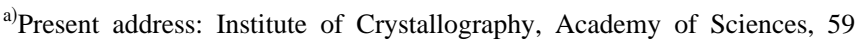
Leninskii prospekt, 117333 Moscow, Russia.

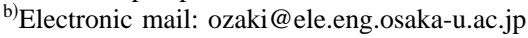

pretilt angle by means of the modulation ellipsometry technique as a function of electric field.

\section{THEORY OF TOTEL INTERNAL REFLECTION (TIR) MODULATION ELLIPSOMETRY}

The terms "modulated ellipsometry"12 or "modulation ellipsometry" 13 are related to the ellipsometric measurement of small changes in optical parameters of a surface induced by the application of an external field (electrical, mechanical, and so on). Here we consider the particular case of modulation ellipsometry for a condition of total internal reflection (TIR) from a uniaxial transparent liquid crystal, when the optical axis of the crystal lies in the incidence plane. In that case the extraordinary $R_{p}$ and ordinary $R_{s}$ reflection coefficients are always equal to 1 . The relation between optical retardation $\sigma=\sigma_{p}-\sigma_{s}$ at the angle of incidence $\varphi$ and the properties of the reflecting surface is defined by Eqs. (1) and (2) as:

$$
\begin{aligned}
& \sigma_{p}=2 \operatorname{arctg} \frac{N \sqrt{N^{2} \sin ^{2} \varphi-n_{\text {eff }}^{2}}}{n_{e} n_{o} \cos \varphi}, \\
& \sigma_{s}=2 \operatorname{arctg} \frac{\sqrt{N^{2} \sin ^{2} \varphi-n_{o}^{2}}}{N \cos \varphi},
\end{aligned}
$$

where $n_{o}$ and $n_{e}$ are the refractive indices of the liquid crystal and $N$ is that of the prism, $\varphi>\arcsin \left(n_{e} / N\right), n_{\text {eff }}$ $=\sqrt{n_{o}^{2} \cos ^{2} \theta+n_{e}^{2} \sin ^{2} \theta}, \theta=\theta_{0}+\delta \theta$, where $\theta_{0}$ is the pretilt angle and $\delta \theta=\delta \theta_{m} \sin \omega t$ is its deviation from the equilibrium state. The Fourier analysis of the experimentally obtained optical retardation $\sigma(t)$ provides a method to calculate the tilt bias angle $\theta_{0}$ and the amplitude of the director oscillation $\delta \theta_{m}$ as follows:

$$
2 \delta(t)=\sigma(t)=A \Delta n_{\text {eff }},
$$




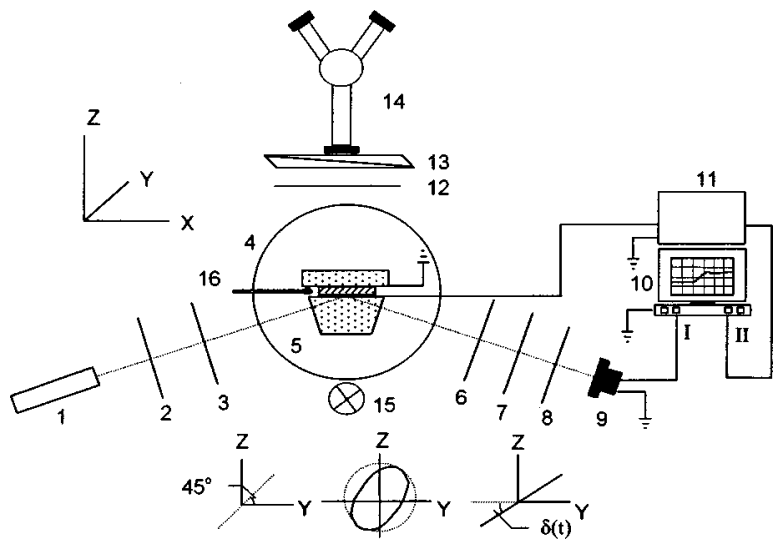

FIG. 1. Experimental setup: (1) $\mathrm{He}-\mathrm{Ne}$ laser, (2) $\lambda / 4$ plate where $\lambda=632.8$ $\mathrm{nm}$, (3) polarizer, (4) rotating stage, (5) cell, (6) $\lambda / 4$ plate, (7) analyzer, (8) filter, (9) PIN photodiode (S1723), (10) IBM compatible computer equipped with sound card, (11) function generator, (12) filter (IRA-05), (13) Glan analyzer, (14) binocular lens, (15) metal-halide lamp (IMN-160), (16) thermometer. (I and II) two inputs of sound card connected to signal and reference inputs.

where $\delta(t)$ is the alternating part of the azimuth angle shown in Fig. 1,

$$
A=\left.\frac{\partial\left(\sigma_{p}-\sigma_{s}\right)}{\partial n_{\mathrm{eff}}}\right|_{n_{\mathrm{eff}}=n_{o}, n_{e}},
$$

and

$$
\Delta n_{\mathrm{eff}}=\frac{n_{e}^{2}-n_{o}^{2}}{2 n_{o}} \theta^{2}
$$

or

$$
\Delta n_{\mathrm{eff}}=\frac{n_{e}^{2}-n_{o}^{2}}{2 n_{e}} \theta^{2},
$$

when the angle $\theta_{0}$ is in the proximity of the $X$ axis $\left(\theta=\theta_{0}\right.$ $\left.+\delta \theta_{m} \sin \omega t\right)$ or the $Y$ axis $\left(\theta=\pi / 2-\theta_{0}-\delta \theta_{m} \sin \omega t\right)$, respectively. Thus we can rewrite Eq. (3) assuming that $\theta$ is small as:

$$
\begin{aligned}
2 \delta(t)=\sigma(t)= & A \frac{n_{e}^{2}-n_{o}^{2}}{n_{o}}\left(\frac{2 \theta_{0}^{2}+\delta \theta_{m}^{2}}{4}+\theta_{0} \delta \theta_{m} \sin \omega t\right. \\
& \left.-\frac{\left(\delta \theta_{m}\right)^{2}}{4} \cos 2 \omega t\right) .
\end{aligned}
$$

The corresponding Fourier components of the electro-optical response can be written as:

$$
\begin{aligned}
& \frac{2 U(\omega)}{\Delta U} \alpha=A \frac{n_{e}^{2}-n_{o}^{2}}{n_{o}} \theta_{0} \delta \theta_{m}, \\
& \frac{2 U(2 \omega)}{\Delta U} \alpha=-A \frac{n_{e}^{2}-n_{o}^{2}}{4 n_{o}}\left(\delta \theta_{m}\right)^{2},
\end{aligned}
$$

where $\Delta U$ is the change in the photodetector voltage, when the analyzer is rotated through angle $\alpha$, and $U(\omega)$ and $U(2 \omega)$ are the sinusoidal amplitudes of the optical modulation at the fundamental and double frequencies, respectively. From Eqs. (5) and (6) we obtain $\theta_{0}$ in the form:
(1)

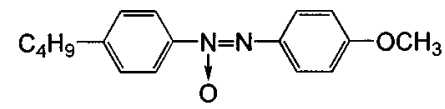

(II)

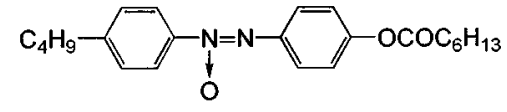

(III)

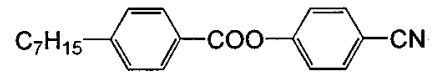

FIG. 2. Liquid crystal mixture: (I) $p$ - $n$-butyl- $p^{\prime}$-methoxyazoxybenzene, (II) $p$ - $n$-butyl- $p^{\prime}$-heptanoyloxyazoxybenzene, (III) $p$-heptyl- $p^{\prime}$-cyanophenylbenzoate.

$$
\theta_{0}=\sqrt{\frac{\alpha}{A \frac{n_{e}^{2}-n_{o}^{2}}{n_{o}}}} \times \frac{U(\omega)}{\sqrt{U(2 \omega) \Delta U}} .
$$

This is the basic equation for the calculation the bias tilt angle $\theta_{0}$ and for the estimation of the accuracy of the technique.

The central point is to excite sinusoidal director oscillations due to the linear flexoelectric coupling between the NLC and an external electric field. For that purpose, it is necessary to eliminate the volume dielectric torque contribution (this is a quadratic on electric field) by choosing a compensated NLC with zero dielectric anisotropy. For this case the expression for the deviation angle $\delta \theta$ has the form: ${ }^{9-11}$

$$
\begin{aligned}
\delta \theta & =\delta \theta_{m} x \exp (i \omega t+i q z+i \phi) \\
& =\frac{\left(e_{1}+e_{3}\right) E_{m} \sin 2 \theta_{0}}{2 K\left(L^{-1}+(1+i) \sqrt{\frac{\omega \eta}{2 K}}+2 i \alpha_{0} \omega\right)} \exp (i \omega+i q z),
\end{aligned}
$$

where $q=(-1+i)(\omega \eta / 2 K)^{1 / 2}, \quad \phi$ is the phase shift, $L$ $=K / W_{s}$ is the conventional extrapolation length defined by the surface anchoring energy $W=(1 / 2) W_{s}\left(\delta \theta_{m}\right)^{2}, e_{1}$ and $e_{3}$ are the flexoelectric coefficients, $K$ is a combination of the bend and splay elastic moduli, $\eta$ is the effective viscosity (approximately equal to the rotational viscosity) and $\alpha_{0}$ is the surface viscosity. ${ }^{10}$

\section{MATERIALS, CELL PREPARATION, SETUP}

All the measurements were carried out for a compensated mixture of (i) $p$ - $n$-butyl- $p^{\prime}$-methoxyazoxybenzene $(\cong 65 \%), \quad$ (ii) $\quad p$ - $n$-butyl- $p^{\prime}$-heptanoyloxyazoxybenzene $(\cong 32.5 \%)$, and (iii) $p$-heptyl-p'-cyanophenylbenzoate $\left(\sim 1.5 \%, \epsilon_{a} \approx 9\right)$ having the clearing point $T_{c}=75^{\circ} \mathrm{C}$ and a small dielectric anisotropy of about $\epsilon_{a} \approx 0.02$. Chemical structures of the compounds are shown in Fig. 2. For the TIR investigations the prism module was prepared as shown in Fig. 3. The high-index prism (TF-10 glass, $N=1.803$ at $\lambda=0.6328 \mu \mathrm{m})$ and the flat plate, both coated with indiumtin-oxide (ITO) transparent electrodes, are used as the cell walls. Cell thickness is $10 \mu \mathrm{m}$. A thin polyimide (PI) film was spin coated onto the clean surface of the glass plate at $3000 \mathrm{rpm}$ for $10-15 \mathrm{~s}$. In the case of the prism one half of 

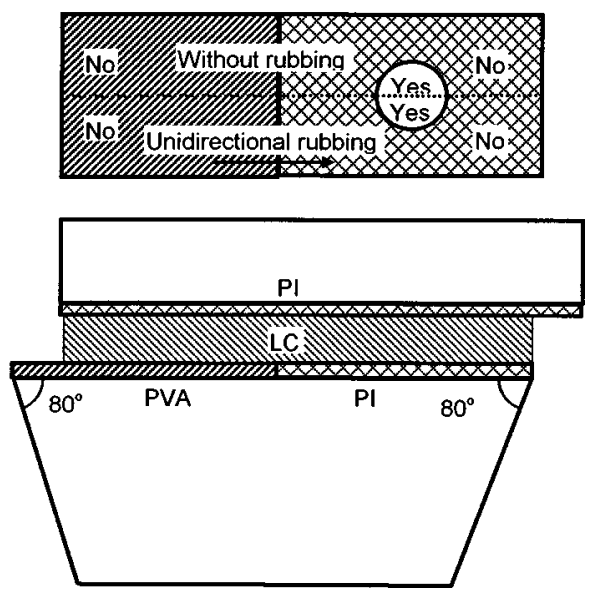

FIG. 3. The aligning treatment of the prism surface.

the working face was covered by PI polymer films by the dipping technique. The thickness of polymer layers ranged from 1 to $1000 \mathrm{~nm}$ depending on the concentration of polymer in the solvent. The aligning films were annealed at $160^{\circ} \mathrm{C}$, to produce thermal imidization and to remove the solvent. To remove the PI layer, the small central region of the PI covered surface was polished by a special paste as shown in Fig. 3. Then half of the prism surface was unidirectionally rubbed with a cloth as also shown in Fig. 3. The prism and the glass plate were assembled into sandwich-type cells with antiparallel rubbed surfaces. The cells were filled at room temperature by placing a drop of NLC at one end of the cell and allowing it to creep through the cell to the other end due to capillary forces. The measurements were always performed within 1 day after filling the cell at room temperature.

The experimental setup is shown in Fig. 1. The cell was installed on an optical bench, and the ellipsometry technique was used to detect the angular oscillations of the nematic orientation at the surface. The principle of our optical modulation ellipsometry method is illustrated by the diagram at the bottom of Fig. 1 showing the successive conversion of the polarization. Within the TIR region a monochromatic linearly polarized beam of a $\mathrm{He}-\mathrm{Ne}$ laser $(\lambda=0.632 \mu \mathrm{m})$ refracted in a heavy glass prism is incident upon the boundary of the LC cell at an angle of $80^{\circ}$ (total reflection angles for uniform homeotropic orientation are about $59.28^{\circ}$ and $79.02^{\circ}$ for the $s$ and $p$ polarizations, respectively, for the investigated NLC). The totally reflected, elliptically polarized beam is analyzed by a quarter wave plate and rotating analyzer. The $\lambda / 4$ wave plate with one of its eigen axes rotated at $45^{\circ}$ from the plane of incidence, converts the elliptical polarization into a linear one. After passing through the analyzer, the light beam is detected by a silicon photodiode. For the signal measurement we have developed a special software based on standard multimedia resources of PC computers to simulate a lock-in amplifier and a fast Fourier signal analyzer.

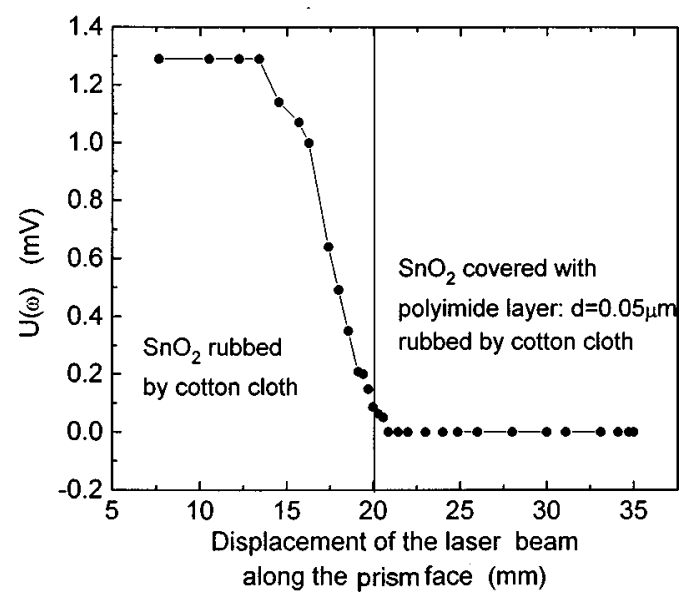

FIG. 4. The surface distribution of flexoelectric response.

\section{EXPERIMENTAL RESULTS AND DISCUSSION}

The reflection of the polarized light from different kinds of interfaces between the NLC and aligning surface layers was studied, as shown in Fig. 3. First of all we would like to underline the fact that changes in phase shift between extraordinary and ordinary beams were not observed for both polyvinyl alcohol (PVA) and PI polymer layers. We also failed to detect any signals for both rubbed and nonrubbed polymer films for the entire thickness range studied. These facts allow us to consider that anchoring energy is sufficiently strong for both rubbed and nonrubbed PVA and PI films, although, e.g., the anchoring strength of an aligning
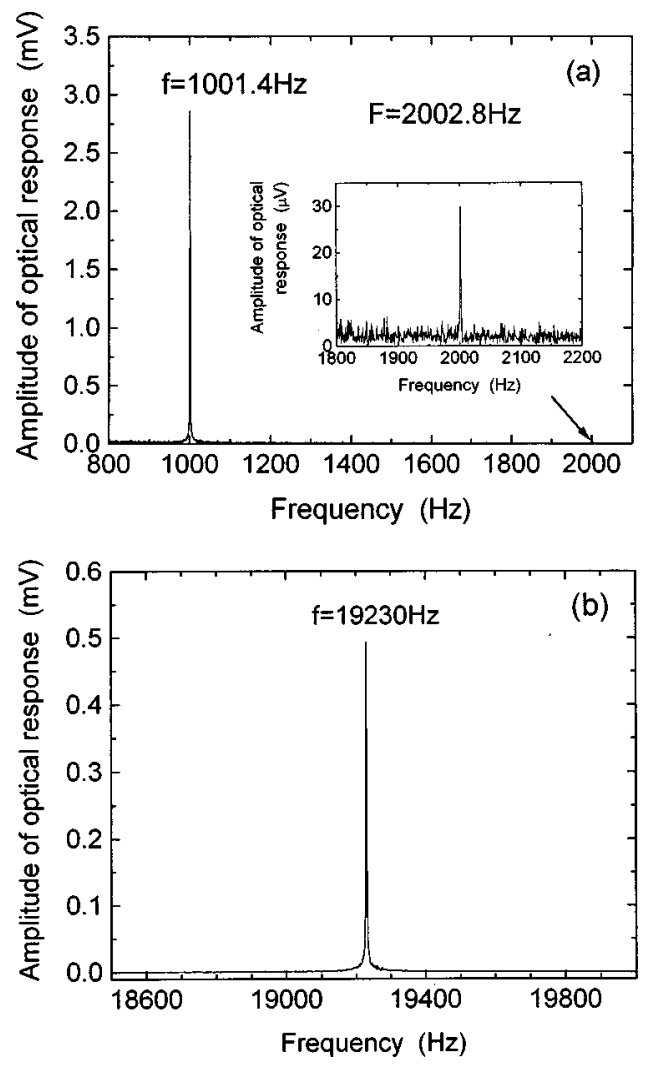

FIG. 5. Fourier transformation of the optical response for excitation frequency: (a) $f=1001.4 \mathrm{~Hz}$, (b) $f=19230 \mathrm{~Hz}$. 


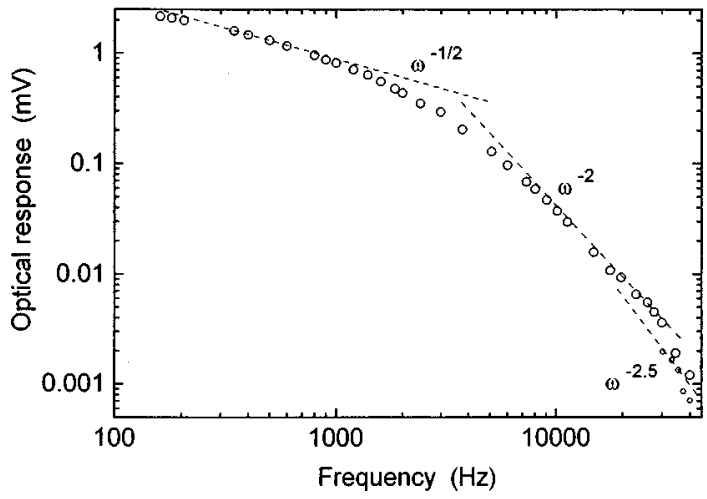

FIG. 6. Amplitude of the first harmonic as a function of frequency obtained using a broad band lock-in amplifier.

nonrubbed film composed of PVA doped with an azo dye was considered to be weak as reported by Iimura $e t a l .{ }^{8}$ The anchoring energy can be easily estimated from Eqs. (6) and (8) taking the static case $\omega=0$, and considering $\theta_{0}$ close to $90^{\circ}$ :

$$
\begin{aligned}
& \delta \theta_{m}>e E \theta_{0} / W_{s}, \\
& \frac{2 U(2 \omega)}{\Delta U} \alpha=-A \frac{n_{e}^{2}-n_{o}^{2}}{4 n_{e}}\left(\delta \theta_{m}\right)^{2},
\end{aligned}
$$

where $e=10^{-11} \mathrm{C} / \mathrm{m}, E=10^{6} \mathrm{~V} / \mathrm{m}, \theta_{0}=0.1, \alpha=5^{\circ}, A=-75$, $U(2 \omega)=5 \times 10^{-6} \mathrm{~V}$ [the noise level shown in the inset of Fig. 5 (a) being equal to $5 \times 10^{-6} \mathrm{~V}$ ], and $\Delta U=0.028 \mathrm{~V}$. From these equations we obtain $W_{s}>5 \times 10^{-4} \mathrm{~N} / \mathrm{m}$ $\left(0.5 \mathrm{erg} / \mathrm{cm}^{2}\right)$ which indicates strong anchoring. ${ }^{2}$

Figure 4 shows the surface distribution of the flexoelectric response obtained by probing with a laser beam along the interface. The curve indicates a marked change in anchoring energy between clean ITO surface and that screened by polymers: high anchoring energy for polymer aligning layers and low for clean ITO electrodes. Further, all our experiments were performed with hybrid cells only, where the clean and rubbed ITO prism face has a weak anchoring energy and the pretilt angle is nearly equal to $90^{\circ}$. The opposite plate covered with the PI layer manifests the homogeneous alignment.

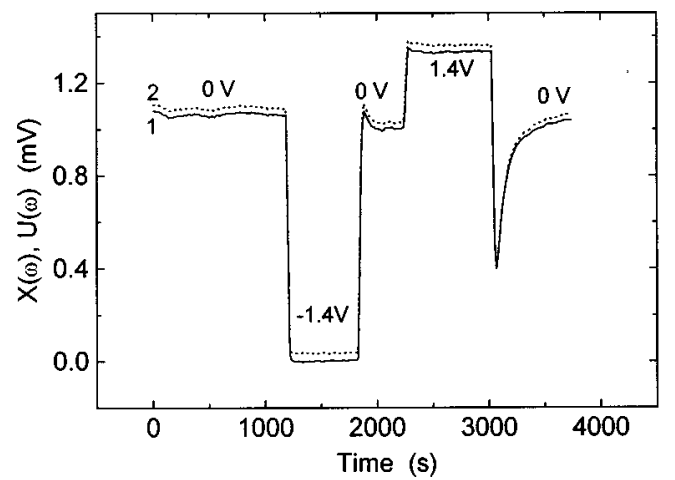

FIG. 7. Time variation of (1) $X(\omega)$ - $x$ component of first harmonic and (2) $U(\omega)$ amplitude of first harmonic of optical response at different dc bias fields measured at room temperature.

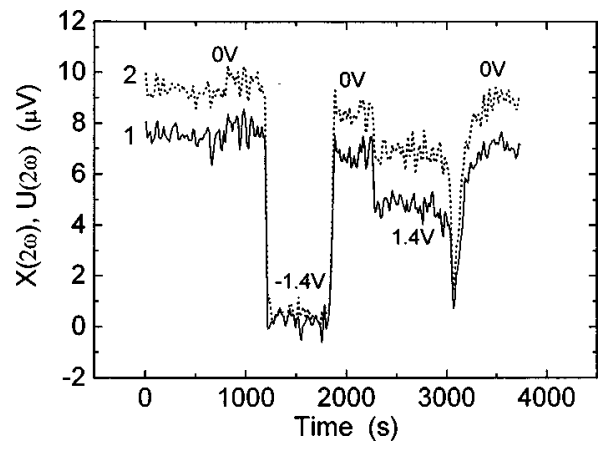

FIG. 8. Time variation of (1) $X(\omega)$ - $x$ component of second harmonic and (2) $U(2 \omega)$ amplitude of second harmonic of the optical response, at different dc bias fields measured at room temperature.

The modulated signal after the photodetector was analyzed by both PhysLab and a lock-in amplifier (Stanford Research Systems, SR-830). Figures 5(a) and (b) present the typical Fourier spectra of the optical signals obtained using the fast Fourier signal analyzer for two different excitation frequencies $f=1001.4$ and $19230 \mathrm{~Hz}$, respectively. The inset of Fig. 5(a) also shows that the second harmonic of the optical response has a smaller amplitude, as compared with the amplitude of the first harmonic. Thus, we could simultaneously measure the amplitudes of the first and second harmonics of the optical response required for the calculation of the pretilt angle. It is worthwhile to note that the experimental error in the determination of the pretilt angle for this case was mostly defined by the accuracy of the determination of the second-harmonic amplitude.

The ac driving voltage applied to the sample had an amplitude of up to $8 \mathrm{~V}$ in order to avoid electrohydrodynamic instabilities (EHDs). The maximum value of the modulation frequency $(22 \mathrm{kHz})$ was limited by the sound card used for the data acquisition process, although the optical response was detectable even at $40 \mathrm{kHz}$ as shown in Fig. 6 . The frequency dependence of the first harmonic of the optical response shown in Fig. 6 has three distinct parts which are governed by different power laws. Such a behavior is correlated with the predictions of Eq. (8) used for the estimation of anchoring energy $W_{s}$, where the alternating part of the pretilt angle has a $\omega^{-1 / 2}$ dependence at low and $\omega^{-1}$ dependence at high frequencies. (The required -2 and

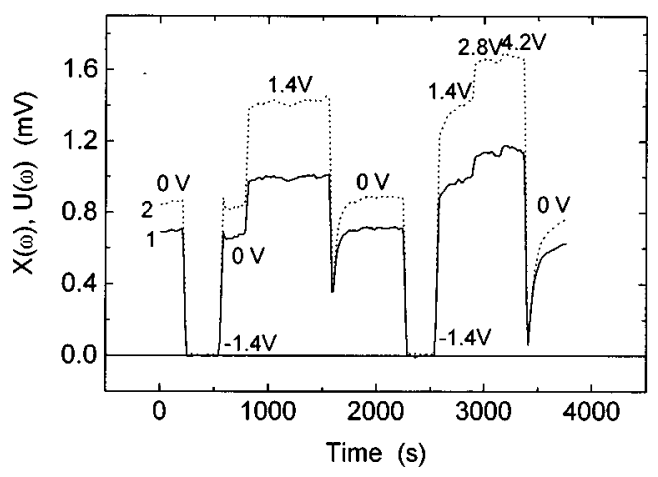

FIG. 9. Time variation of $U(\omega)$ amplitude of first harmonic of the optical response, at different dc bias fields measured at $T=44{ }^{\circ} \mathrm{C}$. 


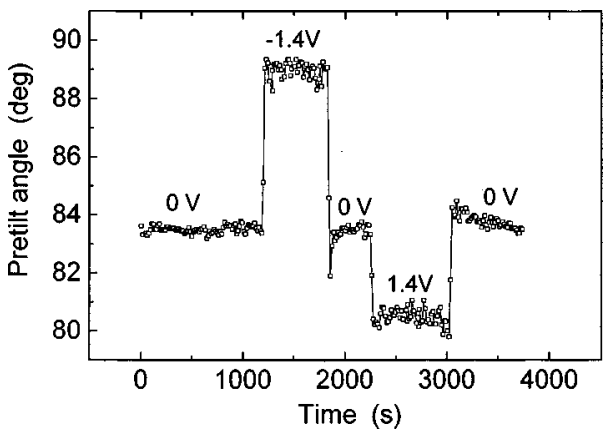

FIG. 10. Time variation of the pretilt angle controlled by dc electric field.

-2.5 powers are derived if we take into account the additional attenuation which is proportional to $\omega^{-1}$ due to the integrating properties of the photodiode circuit and the decrease of the penetration length of the elastic wave which is proportional to $\left.\omega^{-1 / 2}\right) .^{10,11}$

We have recorded the variations of the $X(\omega), X(2 \omega)-x$ components and $U(\omega), U(2 \omega)$ amplitudes of the optical harmonics versus time as shown in Figs. 7 and 8. The deviation of the optical response from the steady state was sensitive to the sign of the dc electric field applied to the LC and manifested sign inversion immediately after switching off the dc bias electric field according to the inversion of direction of the internal electric field. The relaxation times of the transient process were estimated from Fig. 7 to be $\tau_{1}=80 \mathrm{~s}$ and $\tau_{2}=660 \mathrm{~s}$ for switching off negative and positive dc bias fields, respectively. Figure 9 demonstrates the time variation of $U(\omega)$ at an elevated temperature $T=44^{\circ} \mathrm{C}$. In this case the relaxation times decreased to $\tau_{1}=30 \mathrm{~s}$ and $\tau_{2}=370 \mathrm{~s}$. These data are in good agreement with previously reported results where a low frequency $(f=0.005 \mathrm{~Hz})$ phase lag was detected by the TIR modulation ellipsometry technique. ${ }^{10}$ Slow decay $(\tau=14 \mathrm{~s})$ was also observed in dynamic light scattering experiments of porous inorganic matrices impregnated with 4-cyano-4' $-n$-pentylbiphenyl $(5 \mathrm{CB}) .{ }^{14}$ The most plausible explanation for the long relaxation times in the NLC and their sign dependence on the electric field is based on assumptions of the slow formation of a surface layer, ${ }^{14}$ affecting the properties of the electrical double layer and the boundary electric field.

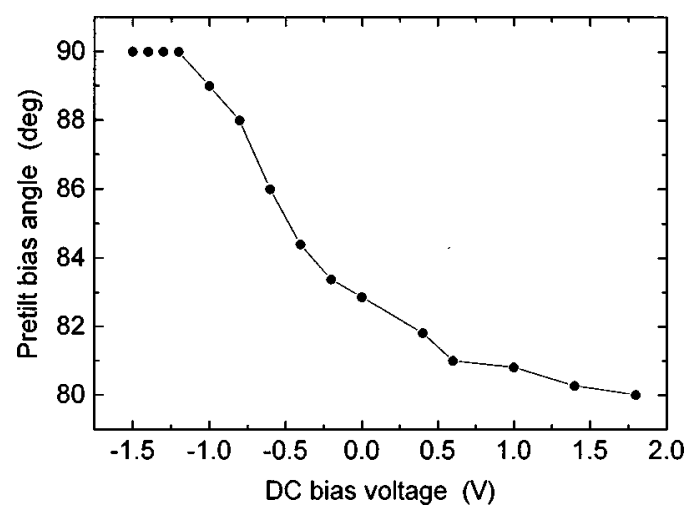

FIG. 11. Ellipsometric measurement of pretilt angle as a function of dc bias electric field.

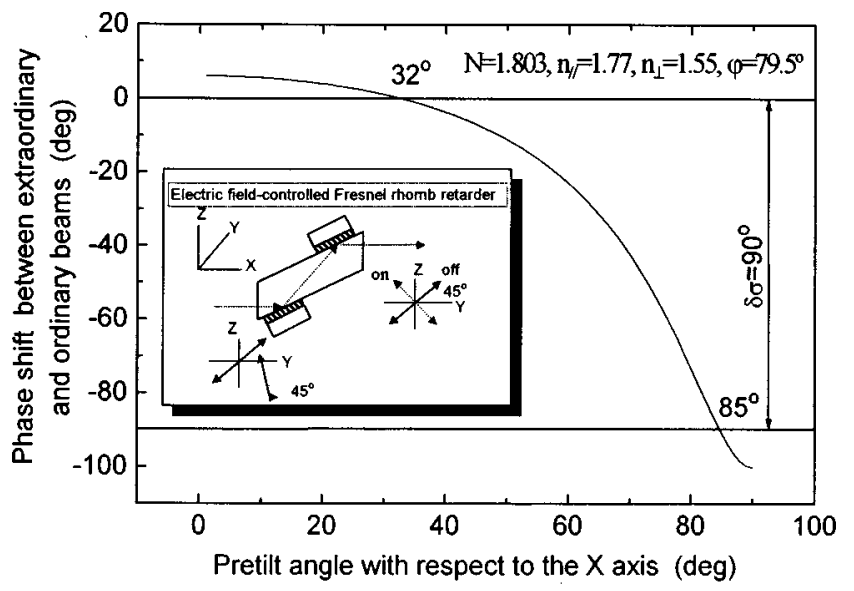

FIG. 12. Electric field-controlled Fresnel rhomb retarder (the inset) and simulated phase shift in the proposed device.

Figure 10 presents the pretilt angles in a hybrid cell versus time at different amplitudes of dc bias field. The amplitudes of first and second harmonics of the optical response were simultaneously measured to calculate the pretilt angle as given by Eq. (7). In the case of the zero bias field, the easy direction was about $83.5^{\circ}$ deviating to about $90^{\circ}$ at $-1.4 \mathrm{~V}$ and $80^{\circ}$ at $1.4 \mathrm{~V}$. Thus, when the electric field is applied to the liquid crystal along the positive $z$ axis, the director points outward away from the normal, while it points toward the cell normal in the opposite case. Such a behavior corresponds to the negative sum of the flexoelectric moduli [as in $N$-(4-methoxybenzylidene)- $4^{\prime}-n$-butylaniline (MBBA).$^{15}$ Figure 11 shows the pretilt angle as a function of applied dc electric field obtained by direct measurement of ellipticity and calculated by Fresnel [Eqs. (1) and (2)]. The analysis of the data in Figs. 10 and 11 shows that dynamic and static measurements of the pretilt angles are in agreement in spite of the different penetration lengths of the elastic and evanescent waves -1 and $5 \mu \mathrm{m}$, respectively.

\section{CONCLUSION}

We have reported the possibility of controlling the pretilt angle based on the flexoelectric effect in a hybrid cell. The large deviation of pretilt angle induced by the dc electric field gives rise to the large optical retardation of the reflected beam which can be used for the controlled Fresnel retarder. Figure 12 shows a simulation result of the phase shift of a proposed Fresnel retarder which has two TIR boundaries having field-controllable liquid crystal layers on their surfaces (shown in the inset).

The designed prism module using a compensated nematic mixture demonstrated a $40 \mathrm{kHz}$ modulation and can be a good candidate for incorporation into waveguiding structures for high speed electro-optical modulators.

\section{ACKNOWLEDGMENTS}

The authors are grateful to M. I. Barnik for supplying compensated NLC. The work was supported by a COPERNICUS grant (Grant No. IC15-CT96-0744), INTAS Grant No. 95-IN-RU-128, and RFFI Grant No. 95-02-0354. 
The work performed at Osaka University was also supported by a Grant-in-Aid for Scientific Research (B) No. 09450143 from the Japan Ministry of Education, Science, Sports and Culture.

${ }^{1}$ K. Kinugava, Y. Kando, and Y. Nagae, Hitachi Rev. 38, 103 (1989).

${ }^{2}$ L. M. Blinov and V. G. Chigrinov, Electrooptic Effects in Liquid Crystal Materials (Springer, Berlin, 1994), p. 180.

${ }^{3}$ T. J. Scheffer and J. Nehring, J. Appl. Phys. 58, 3022 (1985).

${ }^{4}$ M. I. Barnik, V. V. Lazarev, E. E. Maltzev, and N. M. Shtykov, Mol. Mater. 6, 129 (1996).

${ }^{5}$ W. Urbach, M. Boix, and E. Guyon, Appl. Phys. Lett. 25, 479 (1974).

${ }^{6}$ M. Shadt, H. Seiberle, and A. Schuster, Nature (London) 381, 212L (1986).
${ }^{7}$ W. M. Gibbons, P. J. Shannon, S.-T. Sun, and B. J. Swetlin, Nature (London) 351, 49 (1991).

${ }^{8}$ Y. Iimura, J. Kusano, S. Kobayashi, Y. Aoyagi, and T. Sugano, Jpn. J. Appl. Phys., Part 2 32, L93 (1993).

${ }^{9}$ L. M. Blinov, G. Durand, and S. V. Yablonskii, J. Phys. II 2, 1287 (1992).

${ }^{10}$ S. V. Yablonskii, M. Rajteri, C. Oldano, and G. Durand, Proc. SPIE 2731, 87 (1985)

${ }^{11}$ S. V. Yablonskii, L. M. Blinov, A. S. Michailov, S. P. Palto, and L. M. Blinov, Pis'ma v Zh. Teor. Eksp. Fiz. (to be published).

${ }^{12}$ A. B. Buckman and N. M. Bashara, Phys. Rev. 174, 719 (1968).

${ }^{13}$ L. M. Blinov, D. B. Subachyus, and S. V. Yablonskii, J. Phys. II 1, 459 (1991).

${ }^{14}$ F. Aliev, Proc. SPIE 2949, 22 (1997)

${ }^{15}$ G. Barbero, P. Taverna-Valabrega, R. Bartolino, and B. Valenti, Liq. Cryst. 1, 483 (1986). 